\title{
Einstein Proved Wrong, Yet Again
}

\author{
Prabhakar Savargaokar \\ Marine Engineer, Pune, India
}

\begin{abstract}
This short paper is to point out the flawed conclusion drawn on the clocks experiment, performed at the NIST Lab in USA, with rather far reaching implications of toppling Einstein's General Theory of Relativity.
\end{abstract}

Keywords: Clocks, Einstein, Experiment, Labs , Nist

\section{Introduction}

To test Einstein's General Theory of Relativity, a Clocks experiment was performed at the NIST Labs in the USA. This paper questions the conclusions drawn from that experiment.

\section{Description}

Here we are looking at, an experiment performed at NIST Labs USA, in 2010:

https://www.nist.gov/news-events/news/2010/09/nist-pair-aluminum-atomic-clocks-reveal-einsteinsrelativity-personal-scale 2 Atomic/ or optical Clocks were placed, vertically separated by a distance of $33 \mathrm{~cm}$.

The higher clock was reading a faster time and it was concluded that Einstein was right, since according to his GR, time speeds up as we rise above the surface of the earth. But let us take a close look at what is actually happening. Now you are observing 2 atomic/ optical clocks on a lab floor. The higher one shows a higher digital readout. Atomic / optical clocks are nothing but decade counters, to count down the source local oscillator freq. So the higher atomic clock can only show a higher digital readout when its local oscillator freq increases. Here the source oscillator is the Caesium atom/ or oscillating Aluminium ion. Source local oscillator freq can only go up, if time dilates.

( 1 ) Since freq $=$ no of cycles / time and time dilation means you are reducing the denominator, hence higher freq and a higher digital readout.

( 2 ) Alternatively a Vectorial depiction will be as follows. If you stretch the time vector (i.e dilate time ), you can superimpose more no. of cycles on it, hence higher freq and a higher digital readout. Now there is no place to stick any reference frames and Lorentz transformations, by the way.

\section{Conclusion}

Time dilates or slows down and not speed up, as you rise above the surface of the Earth, which is exactly opposite, to that postulated by Einstein's General Relativity. It would be worthwhile to quote here, the physicist Late Richard Feynman ' If your theory, no matter how elegant, does not agree with experiment, it is wrong '. Here the theory in question is Einstein's General Theory of Relativity. 


\section{References}

[1]. Einstein's General Relativity Einstein, Albert (1916). "The Foundation of the General Theory of Relativity" (PDF). Annalen der Physik 354 (7): 769. Bibcode:1916AnP...354..769E.doi:10.1002/andp.19163540702

[2]. Nist Lab Experiment : https://www.nist.gov/news-events/news/2010/09/nist-pair-aluminum- atomic-clocks-revealeinsteins-relativity-personal-scale 